\title{
E SE ANCHE IL CODICE FOSSE UN MESSAGGIO? LA STORIA DEL CODICE HA SENSO SOLO SE IL CODICE NON NE È IL PROTAGONISTA $^{1}$
}

\author{
E SE O CÓDIGO FOSSE TAMBÉM UMA MENSAGEM? \\ A HISTÓRIA DO CÓDIGO SÓ TEM SENTIDO SE O CÓDIGO NÃO FOR O PROTAGONISTA
}

Pio Caroni*

\begin{abstract}
Resumo:
Este artigo conclui pesquisas sobre a história da codificação do direito, que o autor iniciou há meio século. Se até agora tinha dedicado muito (e talvez demasiado) espaço à elaboração histórica de conceitos e, assim, à definição do "estatuto ontológico" do código, aqui prescinde quase totalmente disto. Mas interroga-se, em compensação, sobre o destino concreto enfrentado pelos códigos quando, já como direito vigente, entram na sociedade a que se destinavam e tentam discipliná-la. Vê, por isso, no código uma mensagem, cujo valor (o significado) não se antecipa pelo legislador, mas apura-se pelo destinatário, neste caso, a sociedade. E ela (o esclarecimento não é supérfluo), de onde emergia, a dominava portanto, ou seja, incumbia-se de impor as próprias escolhas de natureza jurídica, social, econômica, política, de sintonizar o código abstrato sobre as suas próprias frequências particulares. Enquanto, até agora, a pesquisa era como que encantada pela história da elaboração/formação do código, esta abordagem diversa desloca o objetivo para além do código, isto é, tenta descrever em quais realidades se encontra o código, uma vez chegado ao seu destino e o que nasce concretamente deste encontro-colisão. E o faz não por desacreditar do que já se fez, mas por descobrir (e descrever) agora a volta completa, graças a uma visão binária, respeitosa tanto da ótica do emitente, quando da do destinatário. A quem interroga propriamente o passado, muitas pesquisas já não interessam, principalmente aquelas que os autores fizeram até agora se movendo exclusivamente a partir do texto sancionado do código. Mas, em compensação, forte na sua visão binária, consegue talvez enriquecer alguns incultos. Ao explicar diferentemente o relacionamento instaurado entre os códigos regulares e irregulares, ao raciocinar de uma forma menos preconcebida sobre a noção de recepção/transplante, ou ao darse conta que - a despeito do que ainda muitos sustentam - cada código modifica, inevitavelmente, muito ou pouco, o quadro da realidade jurídica.
\end{abstract}

Palavras-chave: Emitente (da mensagem). Destinatário (da mensagem). Consolidação/compilação/redação/statement (como produto). $\mathrm{O}$ pós-código. Wirkungsgeschichte (do código). Entstehungsgeschichte (do código). Codificação (como procedimento). Código (como produto). Implementação. Transplante normativo/recepção. Código Civil de 1804.

1 Questo testo, elaborato nell'estate del 2016, fu parzialmente letto in apertura del colloquio a São Paulo il 15 agosto 2016. Una sua versione completa, arricchita di spunti discorsivi emersi durante il colloquio come anche di riflessioni occasionate da successive letture e corredata di note, si potrà leggere nei Quaderni fiorentini per la storia del pensiero giuridico moderno 47, 2018.

* Professore emerito in Storia del Diritto e Diritto Privato della Università di Berna, Svizzera. 


\begin{abstract}
Riassunto:
Questo saggio conclude ricerche sulla storia della codificazione del diritto, che l'autore ha iniziato mezzo secolo fa. Se aveva finora concesso molto (e forse troppo) spazio alla elaborazione storica di concetti e quindi alla definizione dello 'statuto ontologico' del codice, qui ne prescinde quasi totalmente. Ma si interroga in compenso sul destino concreto affrontato dai codici quando, oramai diritto vigente, entrano nella società, alla quale erano destinati, e tentano di disciplinarla. Vede perciò nel codice un messaggio, il cui valore (rispettivamente significato) non viene anticipato dal legislatore, ma via via appurato dal destinatario, in questo caso dalla società. E per essa (la precisazione non è superflua) da chi emergeva, quindi la dominava, ossia da chi era in grado di imporre proprie scelte di natura giuridica, sociale, economica, politica, rispettivamente di sintonizzare l'astratto codice sulle proprie personali frequenze. Mentre finora la ricerca era come stregata dalla storia dell'elaborazione/formazione del codice, questo diverso approccio sposta l'obiettivo sul dopo-codice, tenta cioè di descrivere in quale realtà si imbatte il codice una volta arrivato a destinazione e cosa nasce concretamente da questo incontro-scontro. E lo fa non per screditare quanto già si fece, ma per scoprirne (e descriverne) ora la parabola completa, grazie ad uno sguardo binario, rispettoso tanto dell'ottica del mittente, quanto di quella del destinatario. A chi interroga siffattamente il passato, molte ricerch non interessano più, massime quelle che gli autori hanno finora svolto muovendo esclusivamente dal testo sanzionato dal codice. Gli sembrano virtuali, immaginarie, frammentarie, una traccia sempre più smunta, che poi si perde nella sabbia. Ma in compenso, forte del suo sguardo binario, riesce magari a dissodare qualche incolto. A spiegare diversamente il rapporto istaurato fra codici regolari e irregolari, a ragionare in modo meno preconcetto sulla nozione di recezione/ trapianto, oppure a rendersi conto che - a dispetto di quanto tuttora molti sostengono - ogni codice modifica inevitabilmente, tanto o poco, il quadro della realtà giuridica.
\end{abstract}

Parole chiave: Mittente (del messaggio). Destinatario (del messaggio). Consolidazione/compilazione/redazione/statement (come prodotto). Il dopo-codice. Wirkungsgeschichte (del codice). Entstehungsgeschichte (del codice). Codificazione (come procedura). Codice (come prodotto). Implementazione. Trapianto normativo/recezione. Code Civil 1804.

\title{
1. Introduzione
}

Gli amici che oggi ci regalano questo colloquio - ai quali già per questo motivo diciamo "grazie" - sapevano bene che non conosco a fondo la storia del vostro codice centenario. Se hanno ugualmente desiderato che vi parlassi, e lo facessi per primo, è forse perché vogliono sentirmi ragionare ad alta voce sulla storia dei codici da un punto di vista teorico o, se volete, metodologico. Accolgo questo loro desiderio con gioia, mi incoraggia a proporvi riflessioni recenti e a conferire loro una forma più organica, se non proprio definitiva. Nella speranza che ci aiutino davvero a guardare diversamente alla storia dei codici. 
Insisto su questo fatto: rifletto qui ed oggi su un tema affrontato più volte in passato, anche se in apparenza ho poco da aggiungervi. Non posso riferirvi di recenti clamorose scoperte, che abbiano messo a soqquadro antiche certezze. Non ho scoperto nulla, non ho nulla da rivelare, né nuovi fatti, né nuovi documenti. La mia ambizione è molto più modesta: quella di riordinare fatti già noti, di presentarli diversamente, il che vuol dire incorniciati da un diverso contesto, circondati da un altro entroterra oppure provocati da altre cause, finora inesplorate. Fermo restando che in tutti questi casi questo mio riordinare certezze già acquisire farà riemergere - me lo auguro davvero - evidenze a lungo oscurate o cancellate da un approccio troppo positivistico alla vita dei codici.

Parlo dunque - e lo faccio qui per la prima volta in modo esplicito - del codice come un messaggio e mi propongo dapprima di rispondere a questi interrogativi: Cosa significa vedere nel codice un messaggio? Cosa implica? E cosa provoca?

\section{Essere un messaggio}

Per la teoria della comunicazione messaggio è una notizia, un segno, un testo che un (e)mittente/emissario trasmette/invia/recapita a un destinatario. Un messaggio è per sua natura destinato a viaggiare, a muoversi fra la sorgente e il ricevitore, ad essere consegnato al destinatario. Che provvederà poi ad appurarne il significato, ossia a leggerlo, interpretarlo e dargli se del caso un seguito. Come insegna quell'esperienza quotidiana, che noi regolarmente dimentichiamo: ricorda che il valore/il significato trasmesso dai libri letti, dalle musiche ascoltate, dalle pellicole viste viene appurato dal lettore, dall'ascoltatore, dallo spettatore. Che capiscono/spiegano/interpretano non tanto come vogliono, o devono, o possono, ma come sono.

Se applico questa definizione al codice, cosa succede? Dirò allora:

Il codice, ossia quel testo legislativo che, nell'Ottocento, lo stato elaborò per corrispondere ad attese segnalate dalla società e varò poi in capo a specifiche procedure, non era destinato a restare l'esito felice di un iter parlamentare, un soliloquio autoreferenziale. Il suo destino era diverso, era quello di venir consegnato ad una società, che poco o tanto l'aveva desiderato. Alla quale trasmise perciò non una semplice esortazione, ma qualcosa di più, un programma solo in parte facoltativo, che tendenzialmente la costringeva ad allinearsi. Fermo comunque restando che doveva interpretarlo/realizzarlo nella sua ottica, ossia come prevedevano i suoi programmi e dettavano i suoi bisogni, senza sentirsi condizionata dalle scelte e dalle raccomandazioni del legislatore, ma anche senza ignorarle. Forse si può dire che il codice era un'ossatura senza carne. Spettava perciò alla società prendersi cura di rimpolparla, può ben darsi che lo facesse diversamente da come l'avrebbe fatto il legislatore, se è vero che, come aveva ricordato Portalis in uno dei suoi discorsi preliminari, "les codes se font avec le temps; mais, à proprement parler, on ne les 
fait pas". Ragione per cui può senz'altro capitare che alla fine il messaggio, così arricchito/ manomesso/stravolto dalla società, procedesse in una direzione che il legislatore non aveva potuto anticipare. Lo ha confessato candidamente Raymond Saleilles quando ricordò che durante l'Ottocento il code civil del 1804 era altresì diventato "le code de la bourgeoisie, sans le savoir et sans le vouloir". Dopo aver chiarito cos'è un messaggio elenco qualche tratto caratteristico di questo diverso approccio alla storia della codificazione.

a) Colpisce anzitutto il contesto dichiaratamente sociale di tutta questa storia. La società codificante è presente e interagisce dall'inizio alla fine: dapprima sollecita il codice, poi ne promuove l'elaborazione e finalmente, una volta messo in vigore, lo metabolizza. Non è escluso, ma resta per sua natura difficilmente documentabile, che una siffatta incombenza del sociale abbia talora moderato la tentazione dei giuristi di considerare astrattamente le regole del codice per disporne più facilmente.

b) Alla monomania tipica del positivismo giuridico, cioè alla identificazione /parificazione che esso implicitamente suggeriva fra il testo del codice e la realtà normativa, dietro alla quale si agitava l'autoritarismo statale, la mia proposta di considerare il codice quale messaggio contrappone una verità cristallina. Per essa ogni codice riflette un bipolarismo, caratterizzato da due storie, distinte ma collegate. Quella dell'elaborazione (contrassegnata dall'ottica del mittente) e quella dell'attuazione (all'insegna dell'ottica del destinatario). Due storie, che solo uno sguardo binario riesce a percepire e valutare.

c) Quando si evoca il bipolarismo si allude alla necessità di non trattare separatamente le due storie, ma di considerarle segmenti di una stessa traiettoria, che reclamano perciò di venir percepiti in un'ottica comune. A farne le spese sarà ovviamente la prima storia, quella che descrive l'iter del testo e si conclude sempre trionfalmente, ossia con la sanzione parlamentare. Coltivata a lungo esclusivamente già perché più accessibile a livello documentario, talora gonfiata dalla promessa di ricadute politiche, sarà necessario sdrammatizzarla, massime tenendo conto del taglio interlocutorio delle sue proposte come anche della casualità dei suoi materiali. Come si vedrà.

d) Ne uscirà in compenso alla grande la seconda storia, quella di una società che il codice oramai entrato in vigore non lo subisce quale contraccolpo indigesto ma piuttosto, come dicevo, lo metabolizza. Ossia se ne impadronisce in vista di conferirgli quello specifico taglio, quella specifica misura, che ne renderanno poi più agevole e conforme l'attuazione. Solo questa seconda storia, che ci siamo abituati a chiamare Wirkungsgeschichte, ci parlerà del destino affrontato e vissuto dal codice a contatto con gli antagonismi sociali, ci indicherà quante e quali delle sue previsioni e delle sue speranze si sono avverate, ci permetterà finalmente di valutarne il successo. Non dunque in sequela ad un automatismo, ossia ritenendo sacrosanti i suggerimenti del legislatore o 
indiscutibilmente vere le parole del suo testo, ma appurando caso per caso grado e fattezze della sua conversione/accettazione.

e) A conoscenza dell'importanza di questa seconda storia va poi sottolineata la lungimiranza di tutti quei legislatori (e non furono pochi), che per aver già implicitamente visto nel codice solo un messaggio, giocando astutamente d'anticipo ritennero acquisito il diritto dei destinatari di precisarne il contenuto:

chi dilatando occasionalmente i margini dell'apprezzamento giudiziale, chi offrendo alternative e spronando i destinatari a farvi capo, chi permettendo in via eccezionale di violare una determinata regola o chi finalmente sollecitando istanze inferiori a disciplinare contenziosi locali, per loro natura poco familiari al legislatore. $\mathrm{Fu}$ una strategia generalmente vincente, e non a caso: se da un lato riconosceva la libertà del destinatario vis-à-vis della proposta del codice, d'altro canto ne favoriva un esercizio per così dire disciplinato, "incanalato", cioè tendenzialmente conforme alle speranze e alle previsioni del codificatore.

f) Riassumo con una metafora il significato di queste osservazioni:

nel codice confluiscono due storie, collegate ma distinte. Protagonista delle due non è il codice - come generalmente crediamo, secondo me sbagliandoci - ma la società. Una società che elabora e spedisce prima, una che riceve/applica/implementa poi. Della prima storia sappiamo quasi tutto, della seconda poco o nulla. Se noi nel codice vediamo un messaggio implicitamente valutiamo. Nel senso che premiamo la seconda storia, perché solo essa ci ragguaglia in punto alla realtà del codice. Va perciò affrontata con coraggio. Potrei concludere dicendo: la differenza fra queste due storie è paragonabile a quella fra il biglietto del treno, che acquisto prima di partire, e il viaggio che, grazie a questo biglietto, finalmente affronto.

Come ho ricordato inizialmente: nel passato mi ero più volte avvicinato a questo modo di squadrare la storia, ma non avevo ancora scoperto la forza di questa nuova idea che vede (anche nel codice) un messaggio. E che pensarne ora? In fondo potrebbe trattarsi di un abbaglio, di "un errore provocato da un grosso equivoco", come recita il Dizionario. Appare perciò almeno prudente metterla alla prova, questa affermazione, discuterla affrontando temi tradizionalmente controversi della storia delle codificazioni, vedere se contribuisce a risolverli o complica ulteriormente le cose. Sarebbe troppo volgare chiedere se serve a qualcosa; ma è legittimo sperare che ci permetta almeno di vedere più nitidamente e capire meglio.

\section{Aggrapparsi alle parole}

Iniziamo dunque da quelle storie, e non sono poche - già perché ogni stato che si rispetti ha le sue - che ruotano tutte attorno al testo di un codice così come fu 
varato dall'autorità legislativa e dunque prima che venisse metabolizzato dalla società. A chi le scrive bastano le parole e tutto quanto queste sanno evocare e suggerire. Vi si aggrappano letteralmente, ne esplorano le periferie, ne dissodano i contesti, il che spiega come abbiano potuto studiarle mossi da intenti spesso molto disparati. Come del resto aveva loro suggerito Eugène Guillevic, che proprio in tema di mots incitava a "les laisser parler, mais/sans qu'ils se méfient,/ leur faire dire plus qu'ils ne veulent,/qu'ils ne savent". Mi limito qui a pochi esempi, che documentano altresì la pluralità degli approcci.

a) Taluni storici, e sono tradizionalmente i più numerosi, si sono dedicati alla ricostruzione dell'itinerario di un progetto codificatorio dal momento in cui fu dapprima timidamente ventilato fino alla sanzione regia o all'approvazione parlamentare e all'entrata in vigore. Fra tutte le storie qui ipotizzabili, questa - che è la vera Entstehungsgeschichte - è certamente la più nota e la più frequentata, già perché garantisce allo storico un accesso comodo ad una documentazione esaustiva e facilmente reperibile. Storia vincente per definizione, passa spesso e volentieri sotto silenzio le ragioni dei vinti. Le considera ostacoli da sgomberare per rendere più scorrevole il flusso del racconto, perciò raramente meritevoli di ulteriore riflessione. Ragione per cui ne esce di regola un racconto levigato e scorrevole, quasi una progressione inarrestabile, in tutto e per tutto funzionale all'epilogo, inevitabilmente trionfale. I codici dei nostri paesi furono spesso al centro di 'storie' in tutto e per tutto corrispondenti a questa mia rapida descrizione, perciò non è necessario citare esempli concreti o particolarmente illuminanti.

b) Altri storici, preferibilmente attenti alle ascendenze teoriche $\mathrm{e}$ giusfilosofiche dei codici moderni, hanno ragionato sull'incidenza delle relative tradizioni e tendenze del passato. I più accorti non hanno scomodato le 'ideologie' storiche per farlo e meritano già per questo il mio plauso. Grazie alle loro ricerche ci è oramai diventato familiare il debito contratto dall' istanza codificatrice moderna con l'umanesimo giuridico, l'assolutismo politico, il giusrazionalismo e l'illuminismo giuridico. E ne teniamo regolarmente conto, massime quando siamo tentati di rinchiuderci nell'autoreferenzialità della strategia codificatrice.

c) Altri ancora rincorrono le ascendenze materiali, interrogano cioè il passato alla ricerca dell'origine (ma spesso preferiscono dire delle radici) di istituti e regole, che il codice avrebbe poi inteso confermare. Quando, come ancora nel secolo scorso, il destino di cattedre universitarie poteva dipendere dall'esito di tali ricognizioni, questo approccio era tanto fiorente quanto problematico. I codici venivano allora sezionati senza pietà e storici del diritto di diverso orientamento rivendicavano $i$ frammenti più consistenti, per farne poi oggetto delle loro esplorazioni storiche. Alla fine ognuno spiegava qualcosa della storia del codice, ma nessuno tentava di afferrarne storicamente il tutto. Con quale scorno per lo studente, incapace di metabolizzare esiti tanto frammentari, 
non è difficile immaginare. Oggi, sull'onda della globalizzazione, che tutti prima ci allinea e poi ci inghiotte senza pietà, gli spiriti si sono un po' calmati. Se però l'abitudine di ribadire l'ancoramento storico del codice, ed in questo senso di rivendicarne la storicità, sopravvive, lo dobbiamo alla tenacia di colleghi dediti alla ricerca romanistica. Per molti di loro, come si sa, ogni occasione serve per sottolineare, o magari celebrare, l'indiscussa radice romanistica dei codici civili, massime di quelle sezioni che trattano i diritti obbligatori. Il che suona alle mie orecchie come un'omologazione mostruosa, della quale mi occuperò quando, poco prima di concludere, tenterò di capire se i codici hanno davvero cambiato qualcosa.

d) Ovviamente non mancano gli storici letteralmente sedotti dall'ordine sistematico sotteso al codice e lusingati dalle aperture o conquiste che esso prometteva. Si pensi dunque al modo razionale, logico ed in questo senso nuovo di disporre materiali ora astratti, perché preventivamente sottratti al loro humus prerivoluzionario; alla loro conseguente suddivisione in libri, sezioni, capitoli, paragrafi ed articoli; ai nessi e alle vicendevoli dipendenze che venivano a tal modo evidenziati; come anche all'immagine di fondo così veicolata, quella dell'esattezza (rispettivamente della precisione), ad esempio nella versione suggerita dalla metafora dell'orologio, notoriamente blandita dal sovrano assoluto. Spuntarono perciò storie davvero nuove, poiché finalmente attente a tutto ciò: talune intente a sviscerare il discorso logico dei codici, altre dedite a ragionare sulle scoperte, che la legge dell'interdipendenza permetteva di fare, a discutere le strategie destinate a colmare con certezza matematica le lacune del codice o a spiegare quegli improvvisi ed imbarazzanti silenzi, che il diritto dell'Ancien Régime ancora non conosceva.

e) Meritano finalmente attenzione anche tutti quegli storici, che hanno studiato il testo del codice da un'angolazione giuspolitica e forti dei risultati conseguiti hanno poi formulato apprezzamenti critici. Cito qualche esempio recente, per essere sicuro di non venire frainteso.

Penso dapprima alle analisi di André-Jean Arnaud sul Code del 1804, già perché il suo nome figurava fra le persone, che gli organizzatori di questo nostro incontro intendevano invitare, se la recentissima morte (Natale 2015) non ce l'avesse tragicamente rapito. In una ricerca pionieristica di quarant'anni fa aveva sottoposto il Code ad una lettura strutturalista, sfociata poi in un'aperta denuncia, lo accusò di aver organizzato un 'gioco', che prima o poi avrebbe trasformato l'umanesimo dell'ordine borghese, rivendicato inizialmente, in pura e semplice repressione.

Desidero poi ricordare le numerose ricerche dedicate da Xavier Martin alla prima storia del Code, quella conclusasi con la sanzione finale. Perciò rigidamente imperniate sulle "convictions et intentions des rédacteurs du Code Civil", aspiravano 
a parlarne senza far ricorso a quella mitologia, che molto spesso le aveva in passato accompagnate. Prima o poi tutto ciò propiziò interpretazioni apertamente dissacratorie, come ad esempio quella sul classico tema dell'autonomia della volontà.

Non può mancare, per finire, un accenno esplicito all'assolutismo giuridico apparentemente instaurato dai codici e descritto esemplarmente nell'omonima pubblicazione di Paolo Grossi del 1998. Il termine evoca talune drastiche conseguenze dell'avvenuta codificazione del diritto, che l'autore valutò tutte negativamente. Il codice avrebbe soppiantato il tradizionale pluralismo delle fonti, finora preponderantemente consuetudinarie, con un rigido monismo legalistico. Ossia con una a dir poco soffocante dittatura che umiliava l'interprete, abituato finora a destreggiarsi fra i reperti storici del diritto comune classico, esautorava la società, poiché disconosceva il suo diritto di autogovernarsi, e pretendeva per di più di disciplinare, persino mettere sotto tutela $\mathrm{i}$ privati, quando condizionava la loro autonomia.

Mi fermo qui e provo a concludere su questo terzo punto: ho passato in rassegna un nuvolo di ricerche, quasi tutte recenti, quasi tutte dovute alla penna di validissimi, persino già famosi storici del diritto. Avrei potuto allegarne molte altre. Ma non lo faccio, per non strapazzare la vostra pazienza.

Descrivono storie molto diverse, difficilmente comparabili, questo è vero. Ma hanno ugualmente qualcosa in comune: furono tutte pensate e raccontate da autori ammaliati dal laconismo del testo originale del codice, magari tentati di ritenerlo verorealizzato-implementato ex officio, grazie ad un automatismo tanto fortunato, quanto occulto. Aggrapparvisi, per loro, non era un rischio, ma ribadiva piuttosto una certezza. Chi vede però nel codice un messaggio non può condividerla, questa certezza, la ritiene ingannevole. Sposta infatti l'angolo visuale, punta l'obiettivo sul dopo-codice, guarda oltre, si interroga sul destino al quale andò incontro il codice quando fu inghiottito dalla società, osserva come se lo addomesticò per poi cavarne il servizio desiderato, e come fece tutto ciò naturalmente, senza chiedere il permesso a nessuno e senza bussare preventivamente a nessuna porta.

Le risposte offerte dalle ricerche citate poco fa ci lasciano perciò indifferenti, le riteniamo teoriche, virtuali, immaginarie, esercizi di alta acrobazia lontani da quella realtà sociale, nella quale il diritto è immerso, con la quale interferisce continuamente. Vanno perciò ridimensionate, già perché rispondono a domande, che non sono più le nostre. Non anticipano, non prevedono, men che meno lasciano presagire a quale servizio concreto i codici verranno adibiti, per questo motivo ci lasciano quasi sempre a bocca asciutta. 


\section{Recuperare i codici irregolari}

Ho parlato finora di codici senza peraltro minimamente definirli. Forte di un consenso generale ovvio, quello che ci induce a chiamare codici almeno le leggi così ufficialmente etichettate, quand'anche magari lontane dalle nostre convinzioni teoriche. A nessuno viene in mente di dire che il Codice civile svizzero del 1907 non è un codice, perché manca di una parte generale tradizionale, o che il codice civile brasiliano del 2002 in realtà non lo è, poiché estromette di proposito le norme sulla difesa del consumatore, quindi non comprende tutte le materie giusprivatistiche (come pure dovrebbe).

Nella prospettiva storica ovviamente tutto è più complicato. Perché una storia del concetto di codice c'è ed è importante. E noi non possiamo ignorarla, già perché in essa hanno tradizionalmente pescato gli storici delle singole codificazioni. Con esiti come vedremo - a dir poco discutibili. Questa storia del concetto di codice fu elaborata e scritta da autori che né si conobbero né dialogarono, nei quali noi vediamo tuttavia voci corali. Partendo ad esempio da F. Hotman, senza dimenticare G.W. Leibniz, per poi approdare a Bentham e Savigny. Ovviamente precursori i primi, protagonisti celeberrimi i secondi. Ai primi saremmo debitori di 'prefigurazioni', ai secondi di quello statuto ontologico, nel quale si riverberebbe l'essenza del codice. L'uso del condizionale segnala la mia incredulità: è un bel racconto, questo, ma del tutto immaginario. Già perché i protagonisti vissero in tempi e luoghi diversi e furono indotti a riflettere sul codice da situazioni tutt'altro che comparabili. E quando, eccezionalmente, furono coetanei, come nel caso di Savigny e Bentham, ne discettarono mossi da ambizioni perfettamente contrapposte. Come ritenerli interpreti corali di un dialogo plurisecolare?

Eppure a noi, che veniamo dopo e che spesso non guardiamo per il sottile, questa storia, per irreale che sia - ugualmente serve. Già perché la consideriamo alla stregua di un "grande calderone", nel quale pescare quei tratti peculiari che connotano lo 'statuto ontologico' del codice. Che potrebbero essere ad esempio questi:

--- la completezza in tutte le sue forme, quindi anche l'esclusività;

--- il rifiuto di qualsiasi forma di eterointegrazione;

--- l'ossessiva aspirazione all'unificazione territoriale, sociale e sistematica; da cui sembrò lecito dedurre l'assoluta, indiscutibile centralità del codice.

Si poteva arrivare fino a qui. Magari aggiungere che nella realtà tutto risultava meno lineare e evidente e che comunque dietro a questi severi canoni identificativi si nascondeva pure il desiderio molto concreto di semplificare l'universo giuridico e di favorirne la visibilità. Per concludere che procedendo in questa direzione prima o poi al vecchio sistema delle fonti sarebbe mancato lo spazio. Ma gli storici hanno preferito imboccare un'altra strada e si servirono dello statuto ontologico del codice per distinguere e selezionare; misero da un canto i codici 'veri', veri perché riflettevano la definizione 
canonica - e qui troneggiavano ovviamente i tre codici 'giusnaturalistici' - e relegarono nell'altro tutti quelli che si muovevano in modo vago ed inconcludente nell'area codificatoria, senza mai arrivarne ad una. Tutti indistintamente 'irregolari', perché marcati da "reticenze ed incrinature", trafitti da lacune, bloccati da ritardi. Frutto di malintesi, ignoranza, inesperienza vengono allora impietosamente derubricati ed assegnati ad un limbo costellato di apprendisti o aspiranti, se va bene di precursori.

La gravità di questo impietoso oscuramento della realtà viene documentata dall'elenco di tutto quanto fu estromesso perché irregolare. È foltissimo e già per questo imbarazzante e comprende anzitutto:

--- i progetti e le leggi settecentesche, che un po’ ovunque si accontentavano di riordinare il diritto vigente, ragione per cui non furono quasi mai chiamati codici, ma consolidazioni, compilazioni, redazioni, ecc.;

--- i (molti) codici ottocenteschi che - violando regole generalmente condivise, ma non senza buoni motivi - avevano confermato il ruolo sussidiario del diritto comune, inaugurando così quella che è stata recentemente chiamata la coesistenza del diritto comune e del diritto codificato;

--- $\mathrm{i}$ codici promulgati in costanza di strutture cetuali, che perciò ovvero rinunciavano a generalizzare la soggettività giuridica (il che appare logico), ovvero preferivano proclamarla ugualmente, ben sapendo comunque che per il momento questa bella conquista borghese esisteva solo sulla carta.

--- E per finire quei codici, che chiamerei remissivi, poiché applicabili solo in seconda battuta, quando l'ordinamento locale, che era prevalente, taceva. Un codice perciò sussidiario, come lo fu davvero quello prussiano del 1794 e lo è tuttora quello spagnolo del 1889.

Mi fermo qui, ma potrei anche continuare. Involontariamente ci chiediamo: ma allora chi si salva? Effettivamente si salvano in pochi. Max Weber, che questa domanda se l'era già posta cento anni fa, aveva risposto così: secondo la definizione canonica si salva, ma proprio solo per il rotto della cuffia, il Code Civil del 1804. Riassumo perciò così: dapprima abbiamo definito il codice, poi ci siamo messi a giudicare: questo no, questo si, quello no, e così via. Da cento anni in qua, mi pare. Il gruppo dei promossi resta comunque sparuto, quello degli esclusi in compenso dilaga.

Questa è dunque la storia, che tutti possiamo leggere sui nostri manuali. Una vera ecatombe, mi sembra. Come tutti i racconti canonici, ha anch'essa i suoi lati immaginari: la danza dei precursori, il dialogo fra interlocutori invisibili o assenti, la magica autocomposizione di un mosaico, e altro ancora. Per molto tempo, non posso negarlo, convenne anche a me. Ma dal giorno in cui ho iniziato a vedere nel codice un messaggio non mi convince più, quasi ne provo disagio. $\mathrm{E}$ mi costringe a riaprire un discorso chiuso da tempo. E a partire stavolta non da un concetto ideale di codice, 
del quale mi sono maldestramente innamorato, per descriverne poi l'epifania perfetta, scartando tutto quanto disturba o vi si oppone, $m a$ da una società che aspira a risolvere taluni suoi problemi facendo capo a strategie codificatorie. Strategie che dapprima invoca, più tardi metabolizza. E lo fa generalmente senza pensare alle belle teorie dei giuristi, ma in compenso condizionata dalle sue strutture. A queste urge perciò risalire per capire e spiegare tutto quanto la vecchia teoria aveva connotato negativamente, quando non irriso. Le incrinature, i ritardi, le lacune non sono perciò il frutto dell'inesperienza, della maldestrezza o dell'ignoranza dei codificatori, ma risposte preziose, spie che indicano come ricostruire un iter codificatorio realmente altro. Altro significa qui: non interrotto a metà strada, non interlocutorio, non rischiarato da quanto avvenne anni o decenni più tardi, ma autonomo, come può esserlo l'esito (più o meno ponderato) di una scelta precisa.

Cito un esempio, tanto per chiarire: quello dei codici che fecero tuttora posto al diritto comune (senza specificare qui in quale ruolo preciso). Chi rincorre e celebra le definizioni perfette ne inorridisce e condanna. Cito Bentham: questo amalgama corromperà tutto il codice. Chi guarda invece alla società, intuisce subito: questo 'ibridismo' clamoroso rinvia a sue strutture, svela che sono ancora cetuali, tuttora manovrate da un ceto forense tradizionalmente conservatore. È un'informazione preziosa, permette di capire e impedisce di condannare.

Riassumo e concludo: se le cose stanno così non ha più senso di mettere in riga (tutti) i codici, premiare i migliori e disfarsi degli altri. Tutti hanno uguale dignità, tutti recano l'impronta inconfondibile della società che li ha voluti. Chi desidera capire include e non esclude, recupera e non scarta, già perché, a ben vedere, tutti i codici sono irregolari, ma ognuno a modo suo. Tanto riesce a capire chi nel codice vede un messaggio.

\section{Recepire, raggiare, trasferire}

Il terzo tema vi sarà probabilmente familiare: ruota attorno al destino di quei codici, che non solo vennero metabolizzati dalla 'loro' società, ma pure spiccarono il volo e si insediarono in paesi, ai quali non erano originariamente destinati. E lo fecero talvolta in sequela ad un'imposizione manu militari (come insegnò Napoleone quando ordinò l'introduzione del suo codice in molti paesi conquistati, ad esempio nel Regno d'Italia), altre volte perché attrattivi dal desiderio di una società codificante di appoggiarsi ad un modello, come documenta il destino dello stesso codice del 1804 in molti stati del continente europeo e dell'America latina nel corso dell'Ottocento. Il tema è dunque quello dell'irradiamento provocato dalla circolazione dei modelli codicistici. In questi ultimi decenni è stato rivisitato, rideclinato ed arricchito da molti autori di ispirazione sia sistemica che ermeneutica, il che mi faciliterà il compito e mi permetterà di essere più breve. 
La terminologia, alla quale facciamo capo quando parliamo di queste situazioni, evoca la categoria della recezione, allude perciò ad un contenuto-oggetto, che passa di mano in mano restando comunque inalterato. Proprio come un pacchetto consegnato alla posta, che questa si incarica di recapitare al destinatario così come l'aveva ricevuto. Così concepita la recezione di un codice provocherebbe la trasposizione meccanica e in blocco di uno stesso, invariato ordinamento in uno spazio diverso dal luogo di origine.

Gli autori, che ho appena citati, l'hanno generalmente ritenuta fragile e insufficiente, questa categoria, quando non l'hanno respinta. Preferiscono parlare di trapianto (o trasferimento) normativo, poiché solo così è possibile evidenziare la complessità dell'operazione, l'organicità (ossia la non meccanicità) della trasposizione e del flusso informativo, come anche l'incontrovertibile fatto, che chi riceve non è mai passivo, ma seleziona, valuta, sceglie, un fatto così ben documentato da quella che si può chiamare l'incomparabilità degli esiti. Tutte cose, queste, che mi convengono.

Se però alla metafora medico-biologica del trapianto - che ritengo brillante, ma anche un po' sofisticata - preferisco la mia proposta odierna, perché anche in questo contesto ‘circolatorio' vedo nel codice anzitutto un messaggio, che succede? Quello che a me pare un rovesciamento semantico, in forza del quale tutto quanto finora poteva sembrare eccezionale, temerario o azzardato, tutto quanto veniva concesso a denti stretti e solo in presenza di prove irrefragabili, appare di colpo regolare, normale, scontato. Talune situazioni, certe preferenze risultano allora ovvie, altre invece difficilmente spiegabili, come illustrano questi esempli.

a) Consideriamo dapprima quei paesi, nei quali un codice esterno fu introdotto (rispettivamente adottato) integralmente, per libera decisione dell'autorità politica o in sequela a pressione esterna. Il buon senso sembrerebbe in questi casi suggerire, che chi adotta si allinea, ovvero in ossequio alla scelta operata, ovvero piegandosi all'imposizione. Ma la realtà è d'altro avviso, mostra infatti che persino laddove i codici furono imposti giudici e giuristi li lessero come voleva (o permetteva) la loro precomprensione, come reclamavano i bisogni della loro società, come pretendevano i contesti 'altri', nei quali operavano. L'analisi dell'applicazione dell'ABGB austriaco nel Lombardo-Veneto, come quella del destino del Code Civil nelle terre tedesche lo conferma. Analogamente del resto a quanto in quegli anni avveniva in quegli stati dell'Iberoamerica, ove il riordinamento giuridico /giusprivatistico, così importante per l'autonomia appena conquistata, fu conseguito mediante l'adozione del codice francese. Perché anche qui gli articoli del Code furono ovviamente letti ed interpretati come reclamavano le circostanze locali. 
b) Quando poi, a partire da metà Ottocento, il codice straniero venne sempre più utilizzato come semplice modello? Anche qui può essere utile considerarlo quale messaggio, poiché ribadisce sia il valore intrinseco del modello che la libertà di chi se ne avvale di appoggiarvisi o di distanziarsene, imboccando altre strade. Penso concretamente alla libertà di scegliere e selezionare le norme interessanti secondo le esigenze della vita pratica, di cambiarle, completarle o collegarle ad altre di diversa provenienza, di commentarle e spiegarle come vuole la teoria o reclama l'antagonismo sociale; ma anche a quella di preferire finalmente al modello un'elaborazione del tutto autoctona, nel segno della creatività peraltro insopprimibile del destinatario. Come esemplarmente conferma proprio la parabola del Code 1804 nell'Iberoamerica: adottato dapprima formalmente come si vide -, da metà secolo sempre più solo "guía y orientación", ossia presente "como trasfondo", vieppiù accantonato da elaborazioni endogene di contenuto locale. Con il che alludo esplicitamente al codice peruviano del 1852 e soprattutto a quello cileno del 1855 . Grazie all'eccezionale personalità del suo autore Andrés Bello gli riuscì perfettamente di staccarsi dal modello francese, persino di superarlo e di proporsi a sua volta a modello per altri stati del continente. Non sorprende perciò che gli storici del diritto attenti a questi itinerari abbiano recentemente tentato di riassumerne il significato perorando una "Priorisierung des Lokalen", che nell'ottica della tesi che difendo qui oggi appare ampiamente scontata.

c) E meno ancora sorprende, per finire, quella che poco fa ho chiamata l'incomparabilità degli esiti riscontrabile ovunque si codificò all'ombra dello stesso modello, ma con risultati tutt'altro che univoci. Uno spiacevole scherzo del destino per chi ancora crede alla meccanicità della trasposizione normativa, una banale conferma invece per chi tenga conto della insopprimibile peculiarità delle singole società codificanti e in questo frangente si ricorda dell'algida certezza di Montesquieu: "Les lois doivent être tellement propres au peuple pour lequel elles sont faites, que c'est un très grand hasard si celles d'une nation peuvent convenir à une autre".

\section{Cambiare codificando}

L'ultima domanda è anche quella decisiva, perché vorrebbe chiarire e riassumere tutte le altre. È questa: chi codifica il diritto lo cambia? E in caso di una risposta positiva: $\cos a$ cambia?

Ma anzitutto dobbiamo sapere: a chi va rivolta questa domanda? Al protagonista di questa storia. E chi è il protagonista? La risposta sembra scontata: il codice. L'abbiamo sempre messo al centro delle nostre attenzioni, sembra logico e scontato ritenerlo il protagonista e quindi esaminarlo attentamente, alla ricerca di risposte. Ma talvolta ci imbattiamo in frasi o considerazioni rivelatrici, che gettano luce su un altro lato 
della realtà e ci consigliano prudenza. Mi è ad esempio capitato di leggere che sia il codice brasiliano del 1916 che quello del 2002 arrivarono tardi, quando erano già vecchi; oppure che il BGB tedesco, contrariamente a quanto si pensò finora, arrivò in anticipo; o anche, per finire, che un determinato codice, dopo aver atteso a lungo, finalmente trovò il suo tempo, un po' come la musica di Mahler. Sono frasi rivelatrici, dicevo. Ma di cosa? Del fatto sorprendente - che il tempo, il tempo giusto, quello rimpetto al quale è giustificato parlare di anticipo o ritardo, questo tempo non viene scandito dal codice, ma dalla società. Si dice infatti che un codice arriva in ritardo quando la società si evolve e cresce senza l'aiuto del diritto, autonomamente, percorrendo strade altre, e quando finalmente il legislatore se ne accorge ed interviene deve accontentarsi di legalizzare (così giustificando) quanto è già successo. È quindi la società, che dobbiamo interrogare, se desideriamo ottenere una risposta alla nostra domanda. Perché è lei che lo ha voluto, il codice. E lo ha voluto in vista di impedire certe cose e (soprattutto) di favorirne/agevolarne altre. Ossia per cambiare qualcosa. Già per questo motivo non è necessario:

--- risalire ad Eraclito, che già 2500 anni fa capì "che non ci si bagna mai due volte nella stessa acqua di un fiume"; oppure

--- evocare ascendenze significative, poiché mostrano come spesso la legge fu ritenuta la strada maestra per cambiare (così ad esempiol'assolutismo politico, l'illuminismo giuridico, o l'ideologia dei rivoluzionari francesi);

--- e ricordare che non senza motivo si è potuto parlare di una "lotta per la codificazione"; per intuire che il codice, voluto da una società in effervescenza, era fondamentalmente predestinato a cambiare.

Per sapere poi se l'ha fatto davvero e come, dobbiamo dapprima appurare di cosa aveva bisogno questa società nuova, perché stravolta dalla rivoluzione borghese e pronta a riorganizzarsi secondo un programma economico caratterizzato dalla tendenziale transizione all'economia monetaria, dalla divisione del lavoro e dal favore ora concesso al terzo fattore di produzione, al capitale. A seconda dello spiegarsi di questo programma può essere utile distinguere due fasi.

a) L'inizio possiamo considerarlo in sordina, nel senso che fu dominato dal desiderio di unificare territorialmente e socialmente il diritto privato. Per ovviare sia al dualismo droit écrit-droit coutumier, sia alla struttura cetuale della società di Ancien Régime. "Le code civil et uniforme" era perciò chiamato a superare il primo sanzionando una legislazione uniforme, la seconda generalizzando la soggettività giuridica. Di più per il momento non era necessario disporre. Al massimo poteva servire la soppressione di istituti ancora legati all'economia feudale o la garanzia esplicita di talune libertà reclamate dal nuovo sistema economico. Tutte cose, per ottenere le quali una singola legge poteva anche bastare. Una transizione morbida, pare di capire, più 'transazione' che 
transizione, per citare una notissima riflessione di Portalis; accompagnata dal perdurare di antiche pratiche, dalla consultazione, persino dalla ristampa di opere giuridiche tipiche dell'Ancien Régime, quando non dall'interpretazione del Code quale "loi additionnelle" semplicemente aggiunta (o sovrapposta) all'insieme del diritto finora vigente. C'è stato chi in questa souplesse (o moderazione) iniziale ha voluto leggere un riflesso della nozione originaria, pura, incontaminata di codice, ossia di una nozione non ancora traumatizzata dalla ricerca della completezza formale e materiale. Da parte mia registro questa idealizzazione, ma non la condivido. Credo infatti che le società di questo periodo iniziale si siano accontentate di rincorrere l'unificazione geografica e sociale del diritto privato non perché mancasse un concetto più complesso e sofisticato di codice, ma perché proprio solo di questo avevano bisogno. Presero cioè ciò che in quel momento poteva servire, tutto qui.

b) Ma a partire da metà Ottocento la musica cambiò ovunque e la società fu letteralmente divorata da quegli spiriti, ai quali imprudentemente aveva ammiccato. La rivoluzione industriale da un canto, l'inarrestabile dilatazione di un mercato onnivoro e ubiquitario dall'altro la sconvolsero ab imis, distruggendo anche atavici equilibri, finora bene o male operanti. Grazie all'avvenuta decentralizzazione la concorrenza divenne la molla del nuovo sistema economico. Una concorrenza sfrenata e inevitabile, dunque una cosa piuttosto seria, che faceva gongolare gli uni, ma minacciava (quando non distruggeva) gli altri. Per dirla allora in altre parole: il mercato dilagò a vista d'occhio, contagiò la società, la risucchiò e la sottomise. Quella società, che finora bastava chiamare codificante diventò di fatto la società del mercato. Chiediamoci allora: a quale diritto privato poteva aspirare? E cosa le poteva servire? Desiderò semplicemente venir fiancheggiata da un sistema giusprivatistico corrispondente, perciò speculare, ossia altrettanto esclusivo e totalizzante. Fermo restando che tutto quanto praticato o proposto finora non serviva oramai più. Certamente no il vecchio sistema delle fonti. Il suo approccio sapienziale e l'implicito favore concesso alla consuetudine deludevano l'attesa di certezza, ora più vivace che mai. E il suo millantato universalismo appariva, ad un mondo che si scopriva ora aperto e facilmente percorribile, più démodé che mai. Ma nemmeno tutte quelle strategie alternative potevano essere d'aiuto, che furono frettolosamente escogitate all'ultimo momento per evitare il peggio, rispettivamente per salvare capra e cavoli. Il mercato non le voleva, disprezzava le mezze misure. Aspirava a qualcosa d'altro, non a un rammendo dell'ultima ora. Ad un assetto globale e trasparente del diritto che, nel momento storico, solo il codice era in grado di garantire.

Vien allora da dire: proprio quel codice, che aveva finora cucinato a fuoco lento, si era accontentato di garantire uniformità, aveva persino tollerato - come abbiamo visto - l'ulteriore presenza del diritto comune, proprio questo modesto progetto iniziale, se diversamente sollecitato o interpellato reagì: si mise a crescere, a dilatarsi, a supplire. 
E riverberò così facendo la stessa tensione totalizzante che muoveva il mercato. Diventò così l'epicentro di un nuovo sistema delle fonti, ne capeggiò la gerarchia. Un sistema che, grazie al pluralismo normativo così accettato e ordinato, garantiva esclusività e completezza. Ma non solo: composto da leggi e varato in capo a una procedura trasparente offriva pure una copertura ottimale del fabbisogno giuridico, ossia la reperibilità diuturna di un diritto comunque più sicuro della consuetudine, perché più visibile, prevedibile, calcolabile, accessibile, dietro al quale vegliava ora lo stato, a garantirne l'applicazione. Affermandolo non si vuole anche negare che talvolta solo un intervento mirato del legislatore poteva adeguatamente supplire al fabbisogno giuridico del mercato. Come quando fu necessario legiferare in tema di società commerciali e diritto cambiario, o aggiornare le norme sulle garanzie del credito o sulla proprietà mobiliare. Si intende solo ribadire che queste sporadiche incursioni legislative non erano inerenti alla strategia codificatrice, non la presupponevano, volendo potevano compiersi diversamente, esaurirsi ad esempio nel breve, circoscritto ciclo di una legge.

Indissolubilmente legato alla forma-codice (e in questo senso elemento non facoltativo di una sua possibile definizione) era piuttosto qualcosa d'altro, ossia la pretesa di rispecchiare in tutto e per tutto l'analoga struttura della società cresciuta attorno al mercato, che era notoriamente totalizzante, e lo era sotto molti aspetti. Questo 'servizio', se vogliamo chiamarlo così, solo il codice era in grado di offrirlo e, quello che più conta, di offrirlo restando peraltro invariato/intatto. Bastava infatti squadrarlo diversamente, per fargli dire quanto ora urgeva, per dilatare la valenza di istituti disciplinati da tempo, ma finora confinati in ambiti marginali, quindi per cambiarlo, senza alterarne minimamente il testo. Mettiamo il contratto: non l'ha inventato la società ottocentesca, l'ha ereditato dai tempi classici. Ma solo dopo aver abbattuto tutte quelle pareti, che finora l'avevano penalizzato, fu pronta a generalizzarne l'impiego. Di tutte queste cose si accorge però solo chi, dopo aver scavalcato il testo del codice si mette a braccarne le parole, a rincorrerle per vedere poi come la società giorno dopo giorno, e tenuto conto delle urgenze, alle quali intende ovviare, lentamente le assorbe.

\section{Guardare le cose dall'alto}

Sono in chiusura, come si dice talvolta, perciò alla ricerca di un'immagine forte, di una metafora azzeccata, per concludere bene. Potrebbe essere questa: vedere nel codice un messaggio è impegnativo, ci costringe infatti a spostare continuamente l'obbiettivo, a puntarlo su una società vivente, quindi in movimento, per capire come metabolizza il testo legislativo, cosa finalmente ne fa. È un approccio complesso, ma pagante, è come guardare le cose dall'alto, questa potrebbe essere la metafora che cerco. Guardare le cose dall'alto giova anche allo storico, perché dall'alto coglie più nitidamente 
il movimento delle cose e la dialettica dei conflitti, anche di quelli che si nascondono dietro ai testi legali. Gli sarà perciò facile dedurne che ogni codice, già perché dialoga con la società, ed in questo senso interferisce, rideclina costantemente la realtà giuridica. Chiedere se un codice cambia qualcosa, a queste condizioni, è come chiedere se l'acqua è bagnata.

Per onestà e per dovere di cronaca debbo comunque aggiungere - e questo non è un dettaglio - che né questo modo di guardare alle cose dall'alto, né le certezze che me ne vengono, sono oggi prevalenti. Campeggiano piuttosto voci altre, di quegli autori, che per un motivo o un altro preferiscono non staccarsi dalle parole, non rincorrerle oltre l'orto concluso del codice, che considerano la loro terraferma. Quando ragionano sulla storia del codice approdano ovviamente a convinzioni lontane dalle mie. Che io rispetto, ma non condivido. Ve ne parlo brevemente prima di davvero concludere.

a) Non ci stanno dapprima quei romanisti, che non disdegnano il diritto dei codici moderni, già perché lo considerano un riuscito travaso di verità classiche. Regole sempiterne del diritto classico ne costituirebbero "il fondamento, la sorgente e la radice", a partire dal Code Civil (nel quale uno di loro ha visto recentemente niente di meno che l'apogeo della recezione del diritto romano) fino a quelli varati ieri sera. Stando così le cose non vedono perché mai dovrebbero staccarsi dalle parole. Da parole che ricorrendo invariate nel tempo nutrono per di più la dolce illusione della continuità. Le coccolano perciò le parole, ma (sì, c'è un ma) ne vengono pure ammaliati. Al punto da vedere nei codici null'altro che ius proprium, riconducibile per definizione al diritto comune, e da negar loro, ragionando così, quell' autonomia, che ne faceva finora il tratto connotante. Nell'ottica di questi autori il codice rappresenta perciò proprio solo un episodio, una ulteriore tappa, provvisoriamente l'ultima di un ciclo che noi credevamo concluso, nel quale loro invece tuttora si muovono. Provo disagio davanti a questa giuliva certezza, non posso negarlo. Perché? È presto detto. Perché fa di tante erbe un fascio, ossia omologa improvvidamente cose molto diverse, si avvinghia a parole, che però non vengono risparmiate dal flusso del tempo, in esso piuttosto cambiano costantemente significato; ragione per cui la continuità, che questi autori evocano con fierezza, appare debole e smorta, perché solo omofónica e omógrafa.

b) Ma non ci stanno nemmeno tutti quegli autori, ai quali ho già brevemente accennato, che hanno considerato con eccezionale favore la fase iniziale della codificazione ed hanno poi severamente condannato la seconda. La prima si esaurì - ve ne ricorderete - nell'unificazione geografica e sociale del diritto privato, la seconda si svolse nel segno della completezza/totalità, così utile al mercato. Tanto la prima piacque, tanto dispiacque la seconda, da questi critici ritenuta senza mezzi termini una deriva (che porta cioè lontano dai valori iniziali), frutto spurio di un'ideologia totalizzante, a 
ben vedere emergenza ipertrofica. Sognano di vederla diversamente, proprio solo come un'irritazione marginale e passeggera, rispettivamente come una "semplice increspatura nella corrente", naturalmente nella corrente del vecchio sistema delle fonti, che anche questi critici implicitamente ritengono tuttora operativo. Inizia qui, come nel caso appena esaminato, la corsa al ribasso: tesa a denunciare le lacune del codice, perciò a contestarne l'autonomia, per farlo rifluire prima o poi nell'alveo del diritto comune.

A tutti questi autori, che comunque mi hanno anche insegnato cose importanti, ma che purtroppo si bloccano davanti al testo iniziale, forse perché lo ritengono implicitamente realizzato grazie a qualche automatismo misterioso, che quindi non guardano oltre, perché non ne hanno motivo [e se eccezionalmente guardassero oltre gli si confonde la vista, vedono tutto negativo, perciò una deriva, che loro condannano], bene a loro dico che secondo me si fermano troppo presto, che farebbero bene a ragionare sulla mia proposta. Perché se davvero il codice è un messaggio il nostro sguardo è binario, ossia abbraccia le due cose, contempla sia l'inizio che la sua realizzazione/continuazione nel tempo. Sa che la realtà non è l'inizio, ma quanto succede dopo il varo, quanto se ne fa nel tempo, quanto ne fa la società (con i suoi conflitti e gli interventi più o meno disinteressati delle parti in causa, quindi dei suoi politici, professori, giudici, giuristi, ecc.) quando giorno dopo giorno appura il significato del messaggio. Quanto succede dopo il varo non è necessariamente una deriva, un allontanarsi da valori, ma un realizzarsi di valori. Possiamo criticarla, questa realtà. Ma prima di farlo dovremmo accorgercene, percepirla, descriverla. Anche se spesso è difficile descriverla senza valutarla.

La storia dei codici, se studiata muovendo da questa mia premessa odierna, non può essere storia di concetti, di istituti e di norme (ossia quella storia che gli storici del diritto hanno spesso rivendicato come riserva di caccia), ma è storia di concetti, istituti e norme che vivono sciogliendosi nella società così, come la neve si squaglia sotto il solleone agostano.

São Paulo, agosto de 2016. 